\title{
Effect of surface tension on the growth mode of highly strained InGaAs on GaAs(100)
}

\author{
C. W. Snydera) and B. G. Orr \\ H. M. Randall Laboratory of Physics, The University of Michigan, Ann Arbor, Michigan 48109-1120 \\ H. Munekata \\ IBM T. J. Watson Research Center, Yorktown Heights, New York 10598
}

(Received 5 June 1992; accepted for publication 3 September 1992)

\begin{abstract}
We have investigated the molecular beam epitaxy growth of highly strained InGaAs on $\operatorname{GaAs}(100)$ as a function of the anion to cation flux ratio. Using reflection high energy electron diffraction the evolution of the film morphology is monitored and the surface lattice constant is measured. It is found that the cation to anion flux ratio dramatically affects the growth mode. Under arsenic-rich conditions, growth is characterized by a two-dimensional (2D) to three-dimensional (3D) morphological transformation. However, for cation-stabilized conditions, 3D islanding is completely suppressed, and 2D planar growth is observed. We associate these differences in the growth mode with corresponding changes in the surface tension of the overlayer. A high surface tension stabilizes 2D growth. An analysis which relates surface tension to a critical thickness for the onset of coherent island formation supports this view.
\end{abstract}

In order to achieve optimal performances from strained-layer based electronic and photonic devices, active layers must be defect-free with morphologically and compositionally sharp interfaces. Within this context, an important goal is to be able to control the evolution of the film morphology (i.e., the growth mode). Previous work have shown that the growth dynamics of large latticemismatched $(\gtrsim 2 \%)$ epitaxial films during the initial stages can be understood in terms of a two-dimensional (2D) to three-dimensional (3D) morphological transformation in a dislocation-free film (i.e., Coherent islanding). ${ }^{1-5}$ Models reveal that the relevant physical quantities affecting growth are elastic strain, surface diffusion kinetics, and surface tension. ${ }^{1-4}$ While earlier studies addressed the effects of elastic strain and surface diffusion, ${ }^{1,3}$ in this letter, we reveal the controlling effect surface tension has on the growth mode of highly strained epitaxial layers. Experimental results demonstrating the contrasting growth behavior for molecular beam epitaxy (MBE) grown InGaAs and InAs layers on GaAs (100) substrates under two limiting flux conditions, arsenic rich (stabilized) and cation stabilized, are presented. We propose that the surface tension of the growing film may be varied from a low value (arsenic stabilized) to a high value (cation stabilized) by varying the group V to III flux ratio. Moreover, we argue that the dramatic difference in the growth modes we observe can be attributed to such changes in the surface tension. This view is discussed with regards to an expression relating a critical thickness for coherent island formation to the surface tension, misfit, and surface diffusion length.

The general growth characteristics for highly strained (i.e., misfit $\gtrsim 2 \%$ ) $\operatorname{In}_{x} \mathrm{Ga}_{1-x}$ As films on GaAs (100) have been published previously. ${ }^{6,7}$ Here, we examine the growth mode and, to a lesser extent, strain relaxation of these films as a function of the $\mathrm{As}_{4}$ /cation flux ratio. The investiga-

\footnotetext{
${ }^{a}$ Present address: AT\&T Bell Laboratories, 600 Mountain Ave., Murray Hill, NJ 07974.
}

tions have been carried out in an ultrahigh vacuum MBE system equipped with in situ scanning tunneling microscopy (STM) and reflection high energy electron diffraction (RHEED) ${ }^{8}$ A charge coupled device detection scheme for RHEED analysis was used both to monitor the film morphology and to measure the near-surface lattice relaxation in real time. Although not displayed here, the morphologies which we infer from RHEED have been corroborated by STM. Prior to growing the InGaAs film, a $300-400 \mathrm{~nm}$ nominally undoped GaAs buffer layer was grown at $\sim 570^{\circ} \mathrm{C}$, under arsenic-rich conditions, on a $p^{+}$(zincdoped) on-axis GaAs substrate. Large scale $(1 \times 1 \mu \mathrm{m})$ STM images of these surfaces revealed large atomically flat terraces a few hundred nanometers in lateral extent.

Figures 1 and 2 display the contrasting growth characteristics for films grown under arsenic-rich versus Instabilized conditions, respectively. For both cases the substrate temperature was $500-520^{\circ} \mathrm{C}$. In comparison to InAs versus InAs or $\operatorname{In}_{0.5} \mathrm{Ga}_{0.5} A$ s versus $\operatorname{In}_{0.5} \mathrm{Ga}_{0.5} \mathrm{As}$, the growth characteristics we observe here for $\mathrm{In}_{0.5} \mathrm{Ga}_{0.5} \mathrm{As}$ versus InAs under the two different growth conditions are even more striking. Thus, it matters little that the In composition was not held constant between the two experiments. Prior to epilayer growth, the RHEED pattern displayed the typical $2 \times 4$ streak pattern characteristic of an arsenic-stabilized GaAs(100) surface. Figure 1 displays the data for growth with $\mathrm{As}_{4}$ /cation $\approx 10$. Upon initial deposition, the diffraction pattern changed to a somewhat diffuse, streaked $1 \times 1$. After a few more monolayers (2.8 $\AA / \mathrm{ML})$, it began evolving to the 3D spot pattern shown in Fig. 1(b). This transition was accompanied by an abrupt change in the surface lattice constant, shown in Fig. 1(a), to the bulk $\mathrm{In}_{0.5} \mathrm{Ga}_{0.5}$ As value. As discussed previously, ${ }_{1}$ these characteristics are indicative of a 2D-3D morphological transformation in a dislocation-free film. Figure 2 displays the data for InAs growth with $\mathrm{As}_{4} / \mathrm{In}=1.8$. In less than two monolayers the diffraction pattern transformed from a $2 \times 4$ to a $4 \times 2$ streaked pattern like that shown in Fig. 2(a). This reconstruction is characteristic of an In- 


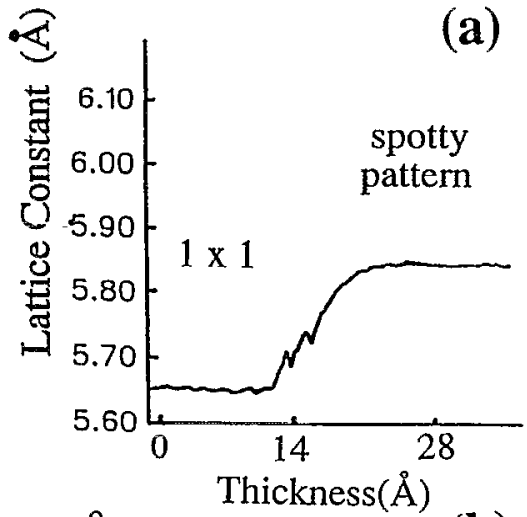

$30 \AA$
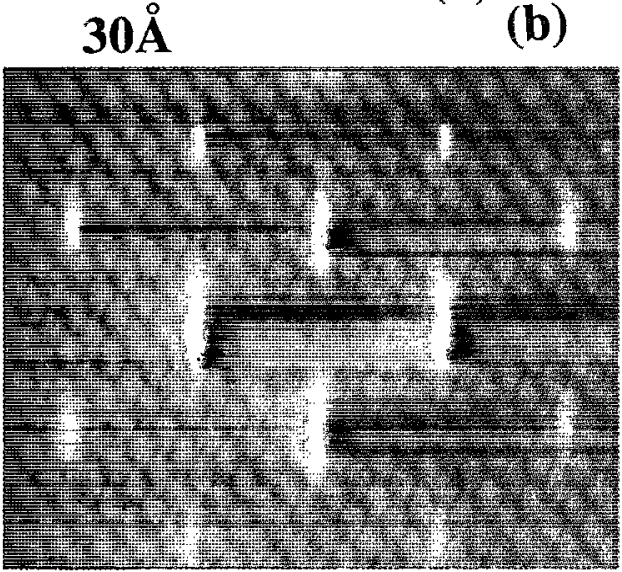

FIG. 1. RHEED data characterizing the growth of $\mathrm{In}_{0.5} \mathrm{Ga}_{0.5} \mathrm{As}$ on $\mathrm{GaAs}(100)$ under As-rich conditions $\left(\mathrm{As}_{4} / \mathrm{In}+\mathrm{Ga} \approx 10\right) ; \phi=[110]$, $T=500-520^{\circ} \mathrm{C}$. (a) Measured surface lattice constant vs thickness. (b) RHEED pattern obtained for a $30 \AA$ thick film. The spot pattern is indicative of $3 \mathrm{D}$ island growth.

stabilized surface and the streaky diffraction features are indicative of a $2 \mathrm{D}$ planar surface. With increasing thickness, the fractional order streaks sharpened and became brighter [see Fig. 2(b)]. The surface lattice constant data of Fig. 2(c) shows that strain relaxed very gradually as the film thickened. We note that films grown with a flux ratio between the As and In-rich limiting values displayed a growth mode which was a combination of those displayed in Figs. 1 and $2 .^{9}$

From the surface lattice constant data, characteristic signatures of the strain relaxation mechanisms are exhibited. When the morphological transformation occurs, coherent islands form and the lattice mismatch is partially accommodated through elastic deformation at the filmsubstrate interface, ${ }^{5}$ and strain is partially relieved via elastic expansion at the free surfaces of the islands. Thereafter, in such a microstructure, barriers to dislocation nucleation and motion are reduced, providing an easy kinetic pathway for further strain relief. The net result is that the observed strain relaxation (lattice constant change) is quite rapid [Fig. 1(a)]. On the other hand, when the surface remains planar (i.e., islanding suppressed), a strict dislocation mechanism operates. Kinetic barriers for dislocation introduction appear to be significant for this situation, consequently strain relaxation is very gradual, occurring over a thickness range of approximately $1000 \AA$ [Fig. 2(c)].
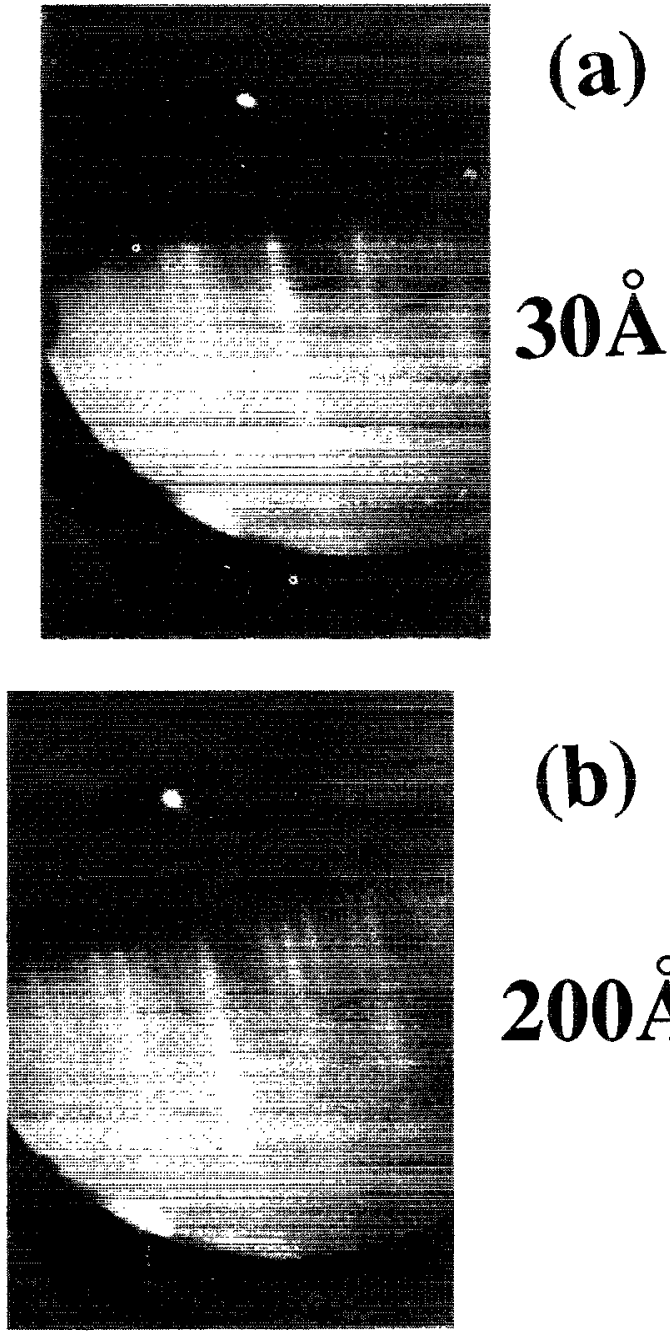

$200 \AA$

(c)

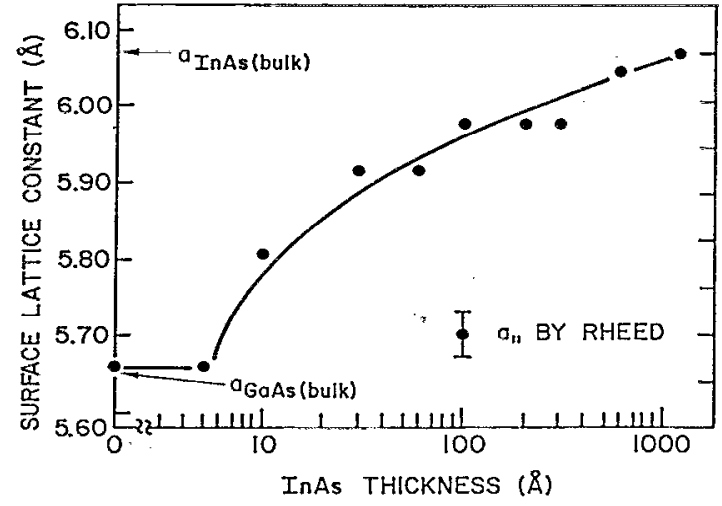

FIG. 2. RHEED data characterizing the growth of InAs on $\mathrm{GaAs}(100)$ under In-stabilized conditions $\left(\mathrm{As}_{4} / \mathrm{In}=1.8\right) ; \phi=[110], T=500-520^{\circ} \mathrm{C}$. (a) RHEED pattern obtained for a $30 \AA$ thick film. (b) RHEED pattern obtained for a $200 \AA$ thick film. The $4 \times 2$ streaked patterns in (a) and (b) are indicative of $2 \mathrm{D}$ growth and an In-terminated surface. (c) Measured surface lattice constant vs thickness.

Focusing now on the growth mode, examination of the diffraction patterns reveals that for arsenic-rich growth there is a transformation from $2 \mathrm{D}$ to $3 \mathrm{D}$ growth after several monolayers have been deposited. This characteristic is quite general in these materials for large misfits (i.e., $\gtrsim 2 \%$ ). ${ }^{6,7,9}$ For example, in the case of InAs(misfit 
$=7.2 \%$ ) deposited under arsenic-rich growth conditions, the $2 \mathrm{D}$ to $3 \mathrm{D}$ transformation occurs at approximately 2 MLs. ${ }^{7}$ Remarkably, for cation stabilized growth at these temperatures the film sustains a $2 \mathrm{D}$ growth mode, independent of misfit.

We propose that the important difference between the As and In tcrminatcd surfaces affecting growth behavior is the surface tension. Previous calculations by Northrup and Chetty on the $\operatorname{GaAs}(\overline{1} \overline{1})$ surface, determined absolute formation energies per $2 \times 2$ unit cell for several structural models. ${ }^{10,11}$ From their calculations it is predicted that the cation-stabilized surface will have a significantly higher surface tension than the As-stabilized surface. While calculations for the (100) surface have not been performed, we expect a similar result. On this surface, as well as others, threefold coordination is topologically rcquired at the surface. Since bulk metallic As is threefold coordinated, energetically it is relatively easy under As-rich conditions to form arsenic surface structures. On the other hand, In or Ga has more of a close packed metallic coordination, thus, in comparison, we expect it to be more energetically costly to form In or Ga surface structures on a cation-terminated surface. $^{12}$

Given that there is such a disparity in the surface tensions of the arsenic and In stabilized surfaces, is it reasonable to expect that it will affect the growth mode in the manner displayed in Figs. 1 and 2? To answer this question, we refer to our previously derived expression for a kinetically controlled critical thickness for coherent islanding, $t_{c}$ (island) $\approx \gamma_{F V}^{2} / \kappa^{2} \epsilon^{4} L{ }^{2,3}$ In comparison to the usual critical thickness for dislocation formation, this critical thickness is for coherent $3 \mathrm{D}$ island formation in a dislocation-free film. It is based on kinetic limitations (to surface diffusion), not energetics. In the expression, $\gamma_{F V}$ is the surface tension of the film, $\epsilon$ is the misfit, $L$ the surface diffusion length, and $\kappa$ is the bulk modulus. Such an expression reflects the physical situation that strained layers grown away from equilibrium (finite $L$ ) need not change to a $3 \mathrm{D}$ growth mode immediately beyond the wetting monolayers (i.e., $t_{c}$ (island) $\neq 0$ ), as is expected based on equilibrium considerations. ${ }^{13-16}$. Additionally, it qualitatively reveals how surface tension and misfit affect the propensity for islanding. Relevant to our discussion here, there is an increase in $t_{c}$ (island) with surface tension. This is intuitively reasonable. Forming an islanded film increases the surface area, thereby costing surface energy. Therefore, with increasing surface tension it is necessary to strain relieve a relatively larger volume (i.e., a thicker film) in order that the energy gain due to a strain reduction in the islands will outweigh the surface energy increase. ${ }^{18}$ Now, however, if $t_{c}$ (island) is greater than the thickness at which it is energetically favorable for misfit dislocations to occur, $t_{c}$ (dislocation), ${ }^{17,18}$ the growing film is expected to remain planar beyond $t_{c}$ (dislocation). Should dislocations form, the strain energy is reduced; consequently the driving force for island formation is diminished. In this way islanding may be suppressed, but strain relaxation will still occur, as shown in Fig. 2. By growing under cation stabilized conditions at lower temperatures than we have here, it is ex- pected that both island formation and strain relaxation (via misfit dislocations) will be inhibited, perhaps enabling rather thick pseudomorphic InAs layers to be grown. It is worth noting that thick, pseudomorphic $\operatorname{In}_{0.5} \mathrm{Ga}_{0.5}$ As layers were previously grown under anion-stabilized conditions at low temperatures. ${ }^{3}$ The low growth temperatures most likely result in an abundance of antisite point defects in the material. However, under cation stabilized conditions, it might be possible to use somewhat higher growth temperatures, producing less-defective layers.

We note that surface diffusion is affected by changing the cation to anion flux ratio, however, it cannot account for the growth mode changes we have presented. Cation surface diffusion is expected to be enhanced by a decreased As flux. ${ }^{19}$ This should lead to an earlier transition to 3D island growth, ${ }^{3}$ not suppress islanding as we have observed.

In conclusion, we have demonstrated that under conditions of imperfect surface diffusion (i.e., finite $L$ ), increasing the surface tension of a strained epitaxial film (in this case InAs on GaAs) will inhibit island formation, thus altering the growth mode. This result contrasts with recent results of surfactant mediated growth, in which it was proposed that lowering the surface tension of strained overlayers was important for suppressing island formation. ${ }^{20,21}$

We would like to thank Dr. M. Chisholm and Dr. S. M. Yalisove for valuable discussions. The work at the University of Michigan was supported by the following grants: DAAL03-88-G-0058, N00014-89-J-1519, NSF/DMR8857828 and the Sloan Foundation. The work at IBM was supported in part by the Army Research Office.

${ }^{1}$ C. W. Snyder, B. G. Orr, D. Kessler, and L. M. Sander, Phys. Rev. Lett. 66, 3032 (1991).

${ }^{2}$ B. G. Orr, D. Kessler, C. W. Snyder, and L. M. Sander, Europhys. Lett. 19, 33 (1992).

${ }^{3}$ C. W. Snyder, J. F. Mansfield, and B. G. Orr, Phys. Rev. B, Oct. 15 (1992).

${ }^{4}$ B. J. Spencer, P. W. Vorhees, and S. H. Davis, Phys. Rev. Lett. 67, 3696 (1991).

${ }^{5}$ D. J. Eaglesham and M. Cerullo, Phys. Rev. Lett. 64, 1943 (1990).

${ }^{6}$ C. W. Snyder, D. Barlett, B. G. Orr, P. K. Bhattacharya, and J. Singh, J. Vac. Sci. Technol. B 9, 2189 (1991).

${ }^{7}$ H. Nakao and T. Yao, Jph. J. Appl. Phys. 28, L352 (1989).

${ }^{8}$ B. G. Orr, C. W. Snyder, and M. Johnson, Rev. Sci. Instrum. 62, 1400 (1991).

${ }^{9}$ H. Munekata, L. L. Chang, and S. C. Woronick, J. Cryst. Growth 81, 237 (1987).

${ }^{10}$ D. K. Biegelsen, R. D. Bringans, J. E. Northrup, and L.-E. Swartz, Phys. Rev. Lett. 65, 452 (1990)

${ }^{11}$ N. Chetty and R. M. Martin, Phys. Rev. B 45, 6089 (1992).

${ }^{12} \mathrm{~J}$. Northrup (private communication).

${ }^{13}$ M. A. Grinfield, Sov. Phys. Dokl. 31, 831 (1986); M. A. Grinfield, IMA Preprint Series \#819 (1991).

${ }^{14}$ M. H. Grabow and G. H. Gilmer, in Initial Stages of Epitaxial Growth, edited by R. Hull, J. M. Gibson, and D. A. Smith, MRS Symposia Proc. No. 94 (Materials Research Society, Pittsburgh, 1987), p. 15.

${ }^{15}$ R. Bruinsma and A. Zangwill, Europhys. Lett. 4, 729 (1987).

${ }^{16}$ D. J. Srolovitz, Acta metall. 37, 621 (1989).

${ }^{17} \mathrm{~J}$. H. van der Merwe and C. A. Ball, in Epitaxial Growth, edited by J. W. Matthews (Academic, New York, 1975), Pt. b, pp. 493-528.

${ }^{18} \mathrm{~J}$. W. Matthews and A. E. Blakeslee, J. Cryst. Growth 27, 118 (1974).

${ }^{19}$ Y. Horikoshi, M. Kawashima, and Hiroshi Yamaguchi, Jpn. J. Appl. Phys. 25, L868_(1986), and references therein.

${ }^{20}$ M. Copel, M. C. Reuter, E. Kaxiras, and R. M. Tromp, Phys. Rev. Lett. 63, 632 (1989).

${ }^{21}$ N._Grandjean, J. Massies, and V. H. Etgens, Phys. Rev. Lett. 69, 796 (1992). 